\title{
EFECTOS DEL FENÓMENO "EL NIÑO 1997-98" SOBRE LOS PRINCIPALES RECURSOS PELÁGICOS EN LA COSTA PERUANA
}

\author{
EFFECTS OF "EL NIÑO 1997-98" PHENOMENON \\ ON MAIN PELAGIC RESOURCES IN PERUVIAN COAST
}

\author{
Miguel Ñiquen, Marilú Bouchon, Sandra Cahuin y José Valdez*
}

\section{RESUMEN}

En el periodo abril 1997 - setiembre 1998 fueron realizados 5 cruceros de evaluación de los recursos pelágicos en la costa peruana, éstos permitieron observar cambios en la composición por especies, distribución, estructura por tamaño, reproducción y niveles poblacionales de los principales recursos pelágicos. Estos cambios guardan similitud con los observados en los eventos El Niño $1972-73$ y 1982-83, especialmente en cuanto a la disminución de la biomasa de anchoveta y el incremento de otras especies pelágicas.

En el período post-Niño se observó cambios notables en la estructura por tallas de anchoveta, mientras que la sardina, samasa y caballa coincidieron en mostrar buenos reclutamientos. La acitvidad reproductiva también se incrementó, inclusive en tallas juveniles, destacando la incidencia de sardinas sexualmente maduras con longitudes de $18-20 \mathrm{~cm}$, situación que no había sido antes registrada.

Palabras claves: Recursos pelágicos, El Niño, costa peruana, poblaciones, pesqueria.

\begin{abstract}
Five evaluation cruises for pelagic resources were performed between April 1997 - September 1998 on Peruvian coast, these allowed to observe changes in composition by species, distribution, size structure, reproduction, and population levels. These changes are similar to those observed in both El Niño 1972-73 and 1982-83. specially with respect to anchovy biomass decrease and increase of other pelagic species.

Considerable changes in anchovy size structure in post-Niño period was observed, while sardine, samasa and Pacific mackerel showed good recruitment. Also the reproductive activity was increased, even in juveniles, with outstanding proliferation of sexual mature sardine with $18.20 \mathrm{~cm}$ length, this situation has not been recorded before.
\end{abstract}

Key words: Pelagic resources, El Niño, Peruvian coast, population, fishery.

\section{INTRODUCCIÓN}

La incidencia del evento "El Niño" es un elemento generador de fuertes cambios en las condiciones oceanográficas del mar peruano, afectando principalmente los recursos pelágicos, produciendo alteraciones en sus procesos biológicos, comportamiento, y una paulatina disminución de sus niveles

*Instituto del Mar del Perú (IMARPE). Callao-Perú poblacionales.

Una de las especies más sensibles a estos cambios es la anchoveta, que en los años 60 alcanzó niveles de biomasa entre 15 y 20 millones de toneladas, rindiendo capturas anuales que llegaron a superar los 10 millones de toneladas en 1970. A partir de 1972 la abundancia del recurso disminuyó drásticamente, debido al efecto combinado de una intensa explotación y las anomalías 
causadas por el fenómeno "El Niño 1972-73" de fuerte intensidad. Esta situación se agravó aún más a inicios de los años 80 con la ocurrencia de otro fenómeno "El Niño" de extraordinaria intensidad en 1982-1983 (Csirke et al., 1996).

El presente trabajo describe el efecto de las anomalías tipo "El Niño" sobre los recursos pelágicos, los que se presentaron en forma fuerte o extraordinaria en 1972-73, 1982-83 y 1997-98, siendo un fenómeno de particular interés para la comunidad científica, especialmente en lo relacionado a cambios en su distribución y concentración, tanto a nivel latitudinal como vertical, así como sus alteraciones de tipo biológico.

\section{MATERIAL Y MÉTODOS}

Las estimaciones de biomasa de los principales recursos pelágicos fueron realizadas utilizando el método hidroacústico, durante las Operaciones Eureka y Cruceros de Evaluación (Tab. 1).

Para el rastreo acústico se empleó ecosonda - ecointegrador SIMRAD, operando a 120 y $38 \mathrm{Khz}$ hasta $250 \mathrm{~m}$ de profundidad.

La información de temperatura y salinidad superficial del mar proviene de la ejecución de estaciones oceanográficas superficiales, cada 10 millas náuticas $(\mathrm{mn})$, durante el desarrollo de las operaciones en el mar y de estaciones costeras en los puertos de Chicama y Chimbote, proporcionadas en el Área de Pronósticos Oceanográficos e Hidrofísica Marina del IMARPE.

En la identificación de las especies se usó las claves de Chirichigno (1978). Se realizaron muestreos biométricos y biológicos de las especies pelágicas en todos los lances de comprobación, con la finalidad de conocer la composición por especies de la captura y las principales características biológicas: estructura por tamaño, peso individual, sexo, madurez sexual.

\section{RESULTADOS}

\section{Condiciones ambientales}

La incidencia del fenómeno "El Niño" se caracteriza por producir calentamientos muy marcados entre Paita y Chimbote (5-10 LS), debido a la penetración de aguas cálidas de tipo ecuatoriales superficiales, con anomalías térmicas extremas de hasta $8^{\circ} \mathrm{C}$ por encima del promedio, en su fase de máximo desarrollo. En cambio, entre Callao e llo, las variaciones son menores y las anomalías alcanzan entre $+6 \mathrm{y}+2^{\circ} \mathrm{C}$.

En general, se conoce que todos los eventos "El Niño" presentan características diferentes (Bjerknes, 1967), sin embargo, por datos de anomalías térmicas en Chicama, tanto en 1971, 1982 y 1996 predominó una etapa fría en el

Tabla 1. Operaciones Eureka y Cruceros de Evaluación.

\begin{tabular}{|c|c|c|}
\hline Nombre del Crucero & Período & Área \\
\hline Op. EUREKA XXIII & 3-6 agosto 1972 & Tumbes ( $3^{\circ}$ LS $)$ - Tacna $\left(18^{\circ} \mathrm{LS}\right)$ \\
\hline Op. EUREKA XXIV & 5-8 setiembre 1972 & Tumbes $\left(3^{\circ} \mathrm{LS}\right)$ - Tacna $\left(18^{\circ} \mathrm{LS}\right)$ \\
\hline Op. EUREKA XXVII & $23-26$ setiembre 1973 & Tumbes ( $3^{\circ}$ LS $)$ - Tacna $\left(18^{\circ}\right.$ LS $)$ \\
\hline BIC HUMBOLDT 8303 & 17 marzo - 3 mayo 1983 & Tumbes $\left(3^{\circ}\right.$ LS $)$ - Tacna $\left(18^{\circ}\right.$ LS $)$ \\
\hline BIC HUMBOLDT 8309 & to setiembre -12 dic. 1983 & Trujillo ( $8^{\circ}$ LS $)$ - Tacna (18 LS) \\
\hline BIC SNP-1 9702-03 & 13 febrero -13 marzo 1997 & Callao $\left(12^{\circ}\right.$ LS $)$ - Tacna $\left(18^{\circ} \mathrm{LS}\right)$ \\
\hline BIC HUMBOLDT 9704 & 4 abril -23 abril 1997 & Paita (5 LS) - Callao (12 $\left.{ }^{\circ} \mathrm{LS}\right)$ \\
\hline BIC HUMBOLDT 9709 & 1 setiembre -11 octubre 1997 & 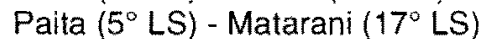 \\
\hline BIC HUMBOLDT 9804 & 20 marzo - 8 mayo 1998 & Tumbes ( $3^{\circ}$ LS) - Tacna $\left(18^{\circ}\right.$ LS) \\
\hline BIC JOSE OLAYA 9806 & 20 mayo - 26 junio 1998 & Máncora $\left(4^{\circ}\right.$ LS $)$ - Tacna $\left(18^{\circ}\right.$ LS $)$ \\
\hline $\begin{array}{l}\text { BIC HUMBOLDT y } \\
\text { BIC OLAYA } 9808\end{array}$ & 20 agosto - 18 setiembre 1998 & Tumbes $\left(3^{\circ}\right.$ LS $)$ - Tacna $\left(18^{\circ}\right.$ LS $)$ \\
\hline
\end{tabular}



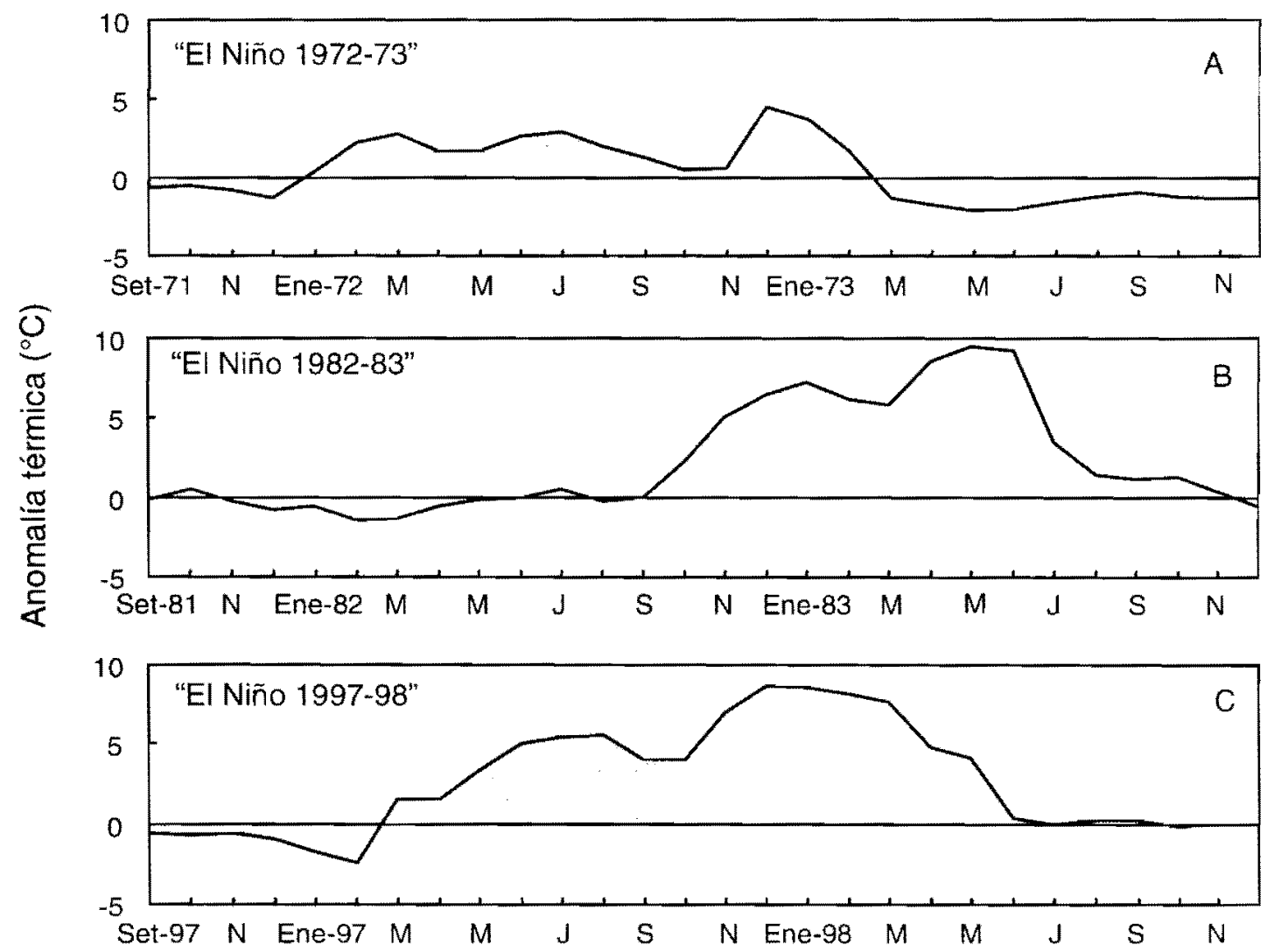

Figura 1. Anomalias térmicas durante eventos "El Niño". Puerto Chicama. A) Calentamiento prolongado. B) Calentamiento corto pero de mayor intensidad. C) Calentamiento prolongado de gran intensidad.

año anterior al inicio del evento, la cual se prolongó, inclusive, hasta los meses iniciales del año siguiente.

El calentamiento observado en 1972 fue muy largo (Zuta et al., 1974), mientras que en 1982-83, fue más corto pero mucho más intenso, en tanto, el calentamiento de 1997 98 fue una combinación de los dos anteriores: largo en el tiempo y fuerte en intensidad. En los 3 casos se produjeron dos picos de desarrollo, siendo la reactivación rápida hacia fines de año y de gran magnitud en el verano siguiente (Fig. 1).

\section{Cambios en las poblaciones de recursos pelágicos}

Según las observaciones realizadas durante períodos "El Niño" se detectó cambios en las poblaciones de los principales recursos pelágicos (Fig. 2).

En la distribución, la anchoveta pasó de una distribución amplia, en toda la costa, a una dispersa y asimétrica hacia el sur del litoral peruano, mientras que la sardina se desplazó de Paita hacia la zona centro-sur, en forma similar a to sucedido en El Niño 1972-73 y 1976 (Zuzunaga, 1985).

En la estructura por tamaños, en el caso de la sardina, de una estructura por tamaños de ejemplares adultos pasó a una estructura mayormente juvenil. En la anchoveta, durante todo el período predominaron los ejemplares adultos, con mínima presencia de juveniles. Solamente en el período post-Niño se incrementó notablemente los pre-reclutas y reclutas de anchoveta. 


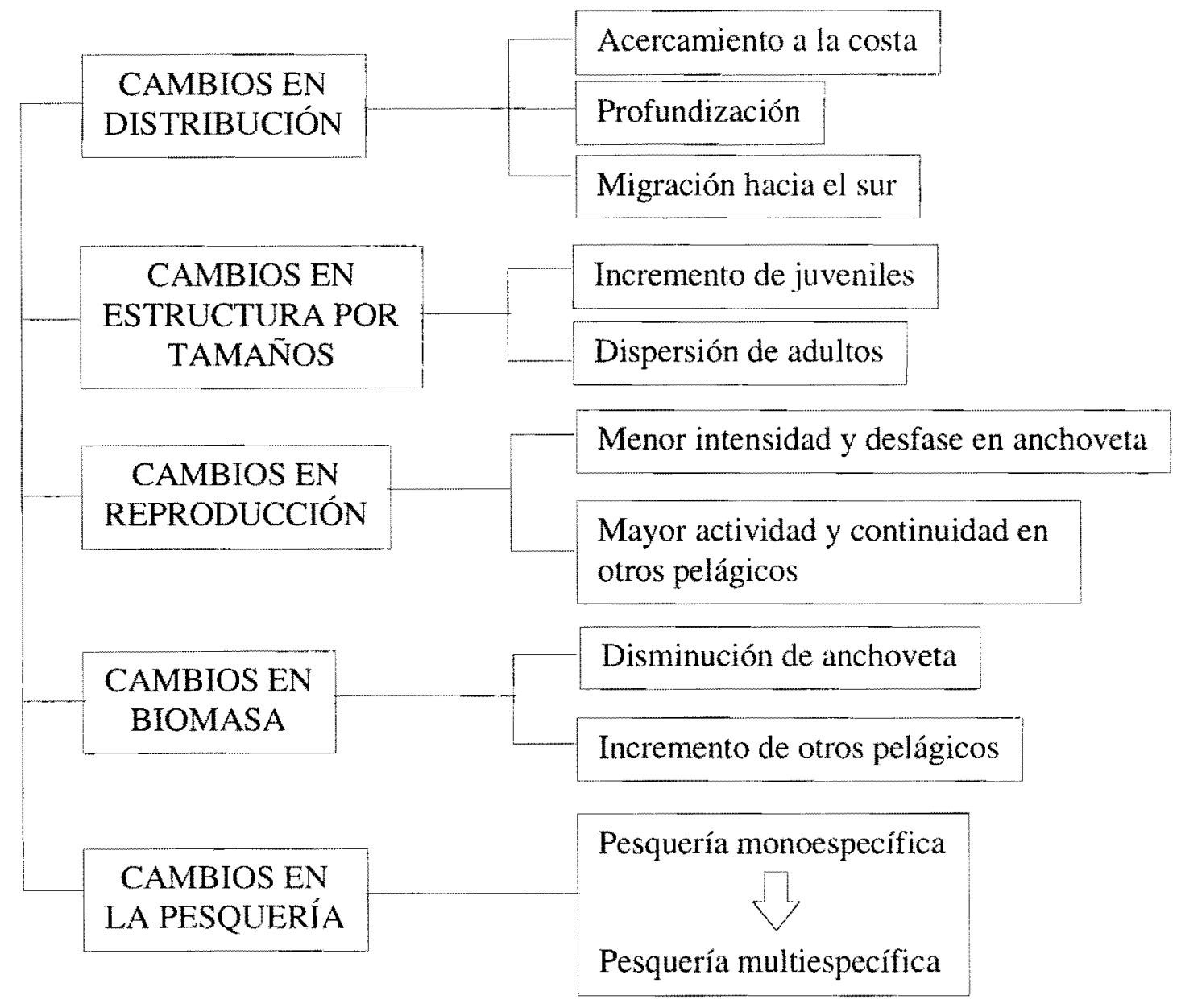

Figura 2. Efectos de "El Niño" sobre los recursos pelágicos.

En el proceso reproductivo, disminuyó la intensidad del desove de la anchoveta e incrementó la actividad reproductiva de otros recursos pelágicos como sardina, jurel, caballa y samasa.

En la estructura de la biomasa de recursos pelágicos, pasó de una predominancia de anchoveta, hacia un predominio de otros pelágicos, tipo sardina, caballa, jurel y samasa.

En la estructura de las capturas, de una pesquería monoespecífica se pasó a una multiespecífica, que incrementó el porcentaje de participación de otros recursos pelágicos y disminuyó la participación de anchoveta, quizás como una protección natural, favorecida por su dispersión tradicional en invierno.

\section{Cambios en la distribución latitudinal y vertical}

Revisando la información de años anteriores podemos describir el desplazamiento de norte a sur y de oeste a este, de varias especies marinas provenientes algunas de ellas de la Provincia Panameña, que por efecto de "El Niño 1997-98" llegaron a tener una presencia constante en la región norte-centro de nuestro litoral. Este desplazamiento puede considerarse como el movimiento de un sistema con sus especies integrantes, las cuales fueron avanzando conforme se intensificaba la anomalía térmica. 

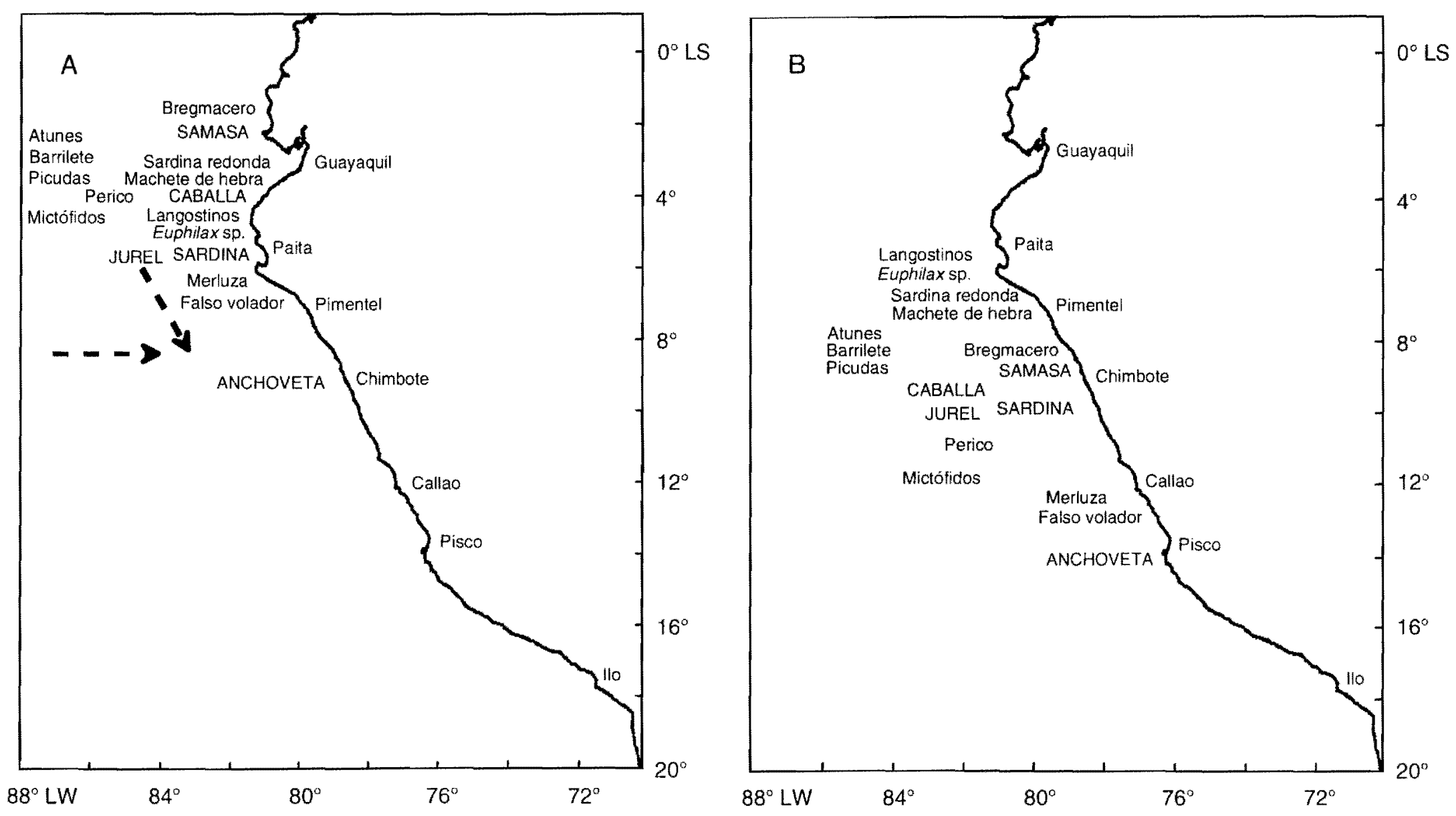

Figura 3. Desplazamiento de especies durante "El Niño 1997-98" en la costa peruana. A) Abril 1997 (inicio de EN). B) Abril-mayo 1998 (final de EN). 

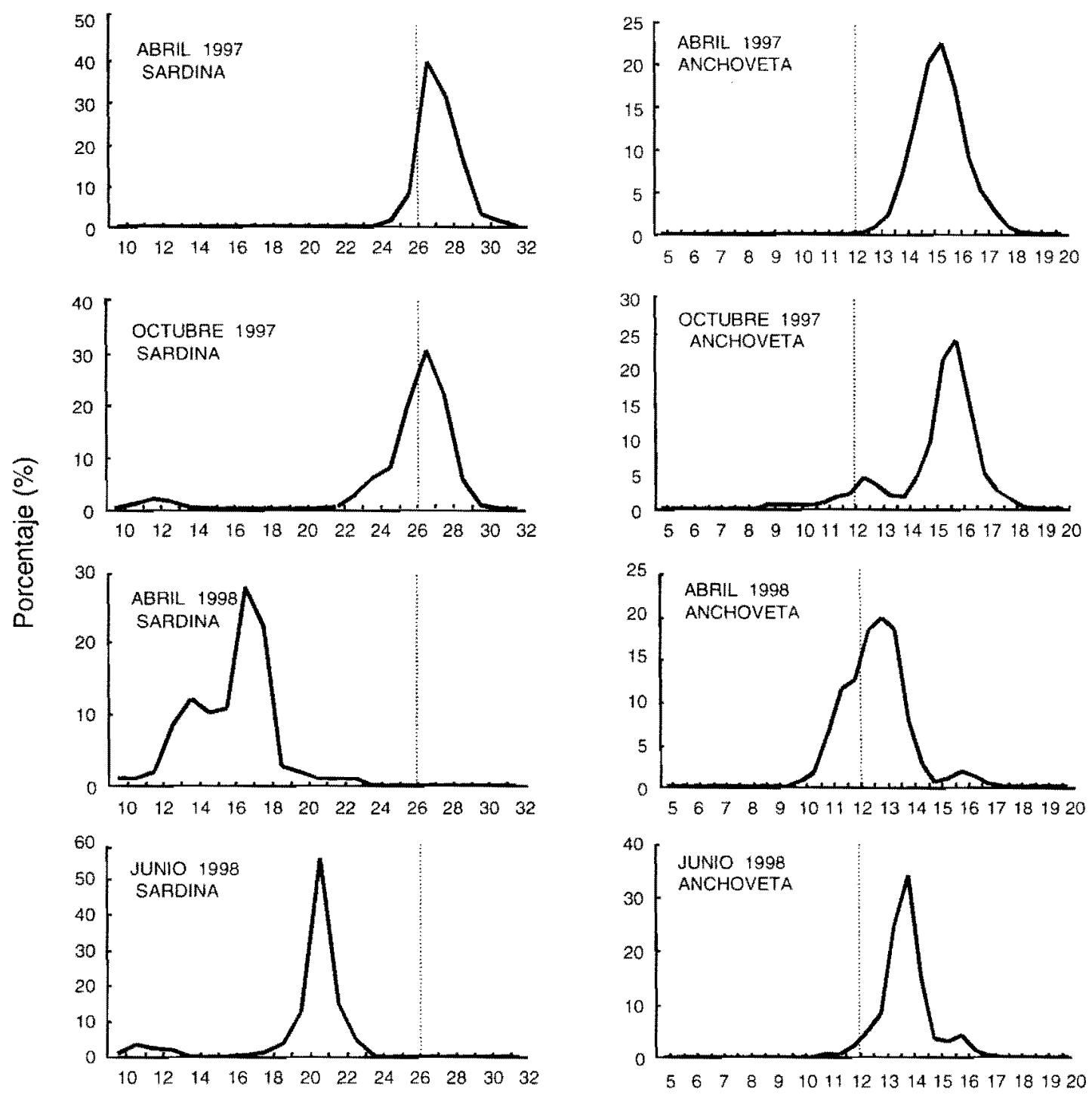

Longitud $(\mathrm{cm})$

Figura 4. Variaciones en la distribución y estructuras por tallas de anchoveta y sardina durante abril 1997 - junio 1998 (línea punteada separa las tallas de adultos y juveniles).

En primera instancia hubo un ingreso intenso de perico (Coryphaena hippuras), atunes (Thunnus spp.), picudas (Sphyraena ensis) y caballa (Scomber japonicus), luego ingresó la samasa (Anchoa nasus), machete de hebra (Opistonema libertate), langostinos (Penaeus spp., Xiphopenaeus riveti), barriletes (Katsuwonus pelamis), y finalmente se detectó el ingreso de mictófidos, bregmacero (Bregmaceros bathymaster, especie de distribución restringida que normalmente se le encuentra frente al Golfo de Panamá), (Chirichigno, 1978); también lo hizo ayamarca (Cetengraulis mysticetus), jurel fino (Decapterus macrosoma), jurel ojo grande (Caranx hippos), pez cinta (Desmodema polysticta) y el cangrejo Euphilax sp. (Fig. 3A).

La incidencia de las condiciones cálidas en la región norte-centro en las etapas iniciales de "El Niño", determinó el repliegue de la anchoveta, en primera instancia hacia la costa, 
a la franja costera de las 20 millas y en altas concentraciones, que la hizo muy vulnerable a la acción de la flota pesquera. Casi inmediatamente las mejores concentraciones empiezaron a desplazarse hacia el sur de Chimbote y también se pudo detectar una profundización de los cardúmenes de anchoveta en la región norte-centro, ubicándose por debajo de los $10 \mathrm{~m}$ y llegando hasta los $60 \mathrm{~m}$ en los puertos de Chimbote $y$ Callao en la fase culminante del evento, este comportamiento determinó un cambio en la estrategia de pesca, donde las mayores capturas fueron en horas nocturnas. En el caso de la sardina, de una distribución normal frente a Paita, sus mayores concentraciones empezaron a trasladarse hacia Pimentel y Chicama, simultáneamente con un acercamiento a la costa. En la fase de mayor impacto su distribución alcanzó hasta llo en la región sur, con mayores concentraciones entre Chicama y Chimbote, ocupando áreas costeras (Figs. 3B y 4).

Un aspecto complementario en la
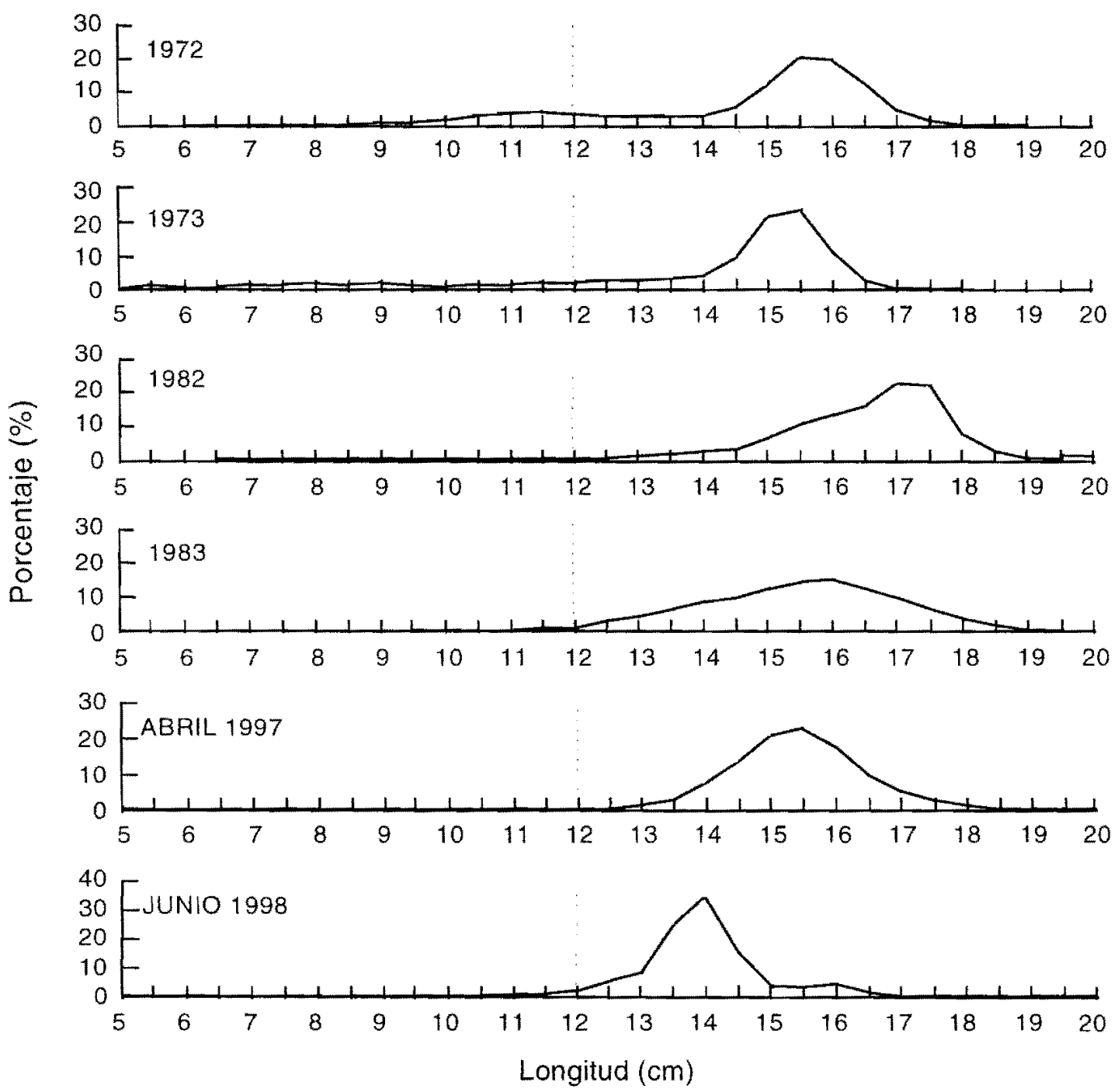

Figura 5. Estructura por tamaños de anchoveta en eventos "El Niño" (linea punteada separa las tallas de adultos y juveniles). 
distribución y disponibilidad de anchoveta fue el comportamiento de las aves guaneras, que a partir de abril de 1997 abandonaron las islas del norte, localizándose principalmente en las zonas central y sur (IMARPE, 1997).

\section{Cambios en la estructura por tamaños}

Durante los eventos El Niño 1972-73, 1982-83 y 1997-98, se observó como un comportamiento general, el predominio de ejemplares adultos y la disminución o ausencia del reclutamiento de anchoveta (Fig. 5), lo que incidió negativamente en la renovación y crecimiento de la población. Sin embargo, a inicios del período post-Niño este comportamiento varió notablemente, con el fuerte ingreso de pre-reclutas y reclutas, (IMARPE, 1998), provenientes de desoves iniciados a fines de "El Niño", y la desaparición de adultos, que probablemente se habian dispersado o replegado muy cerca de la costa.

La sardina (Sardinops sagax sagax) tuvo un incremento de ejemplares juveniles (Fig. 6), principalmente de uno y dos años de edad, en forma continua durante todo el período "El Niño", favorecidos por la presencia de condiciones cálidas, lo que determinó el aumento de su nivel poblacional.

Es importante destacar la sincronía existente entre los buenos reclutamientos observados en las especies sardina, samasa, caballa, y también en merluza (Merluccitus gayi peruanus), falso volador (Prionotus stephanophrys) y también los peces linterna del género Vinciguerria, todos ellos
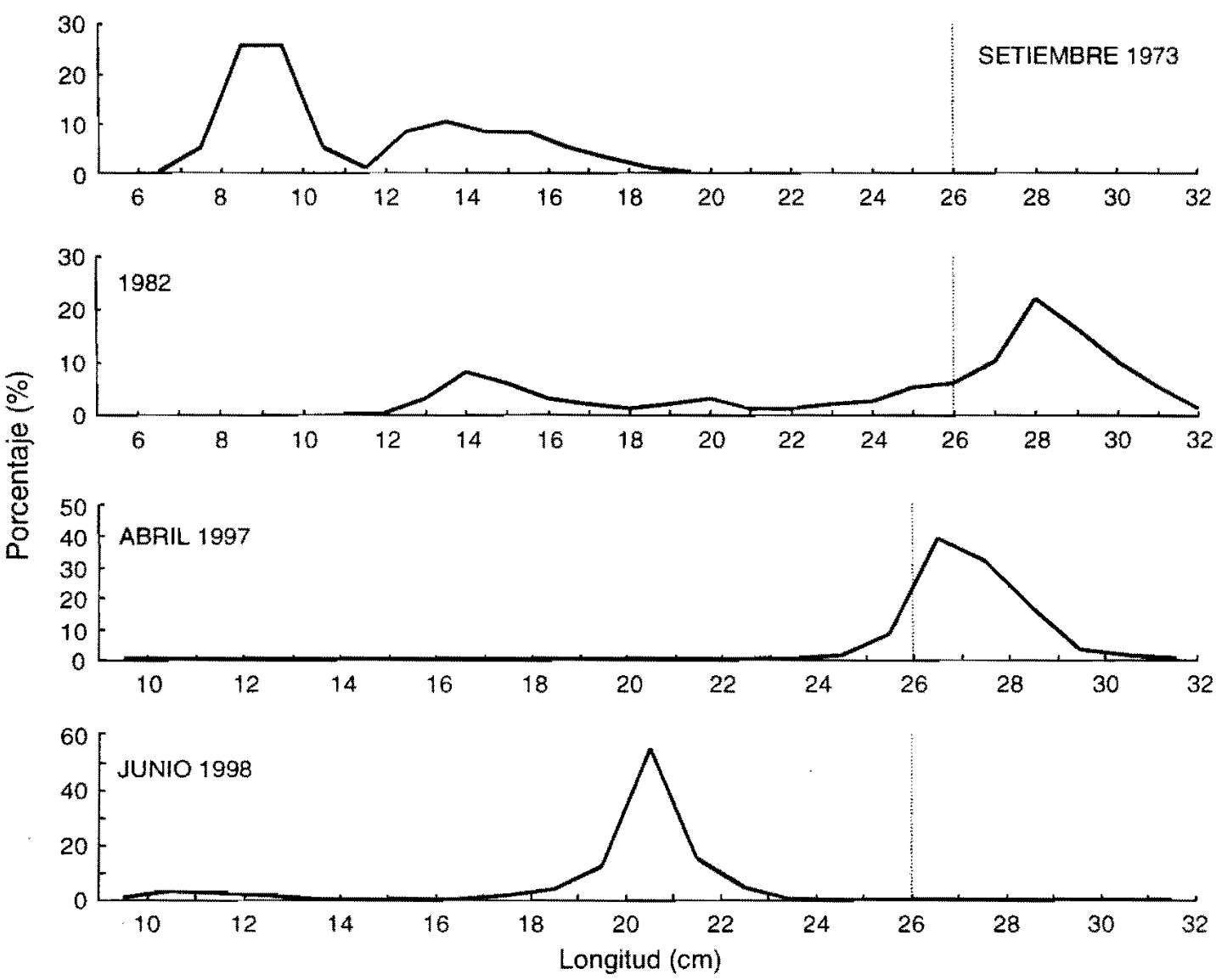

Figura 6. Estructura por tamaños de sardina según eventos "El Niño" (línea punteada separa las tallas de adultos y juveniles). 


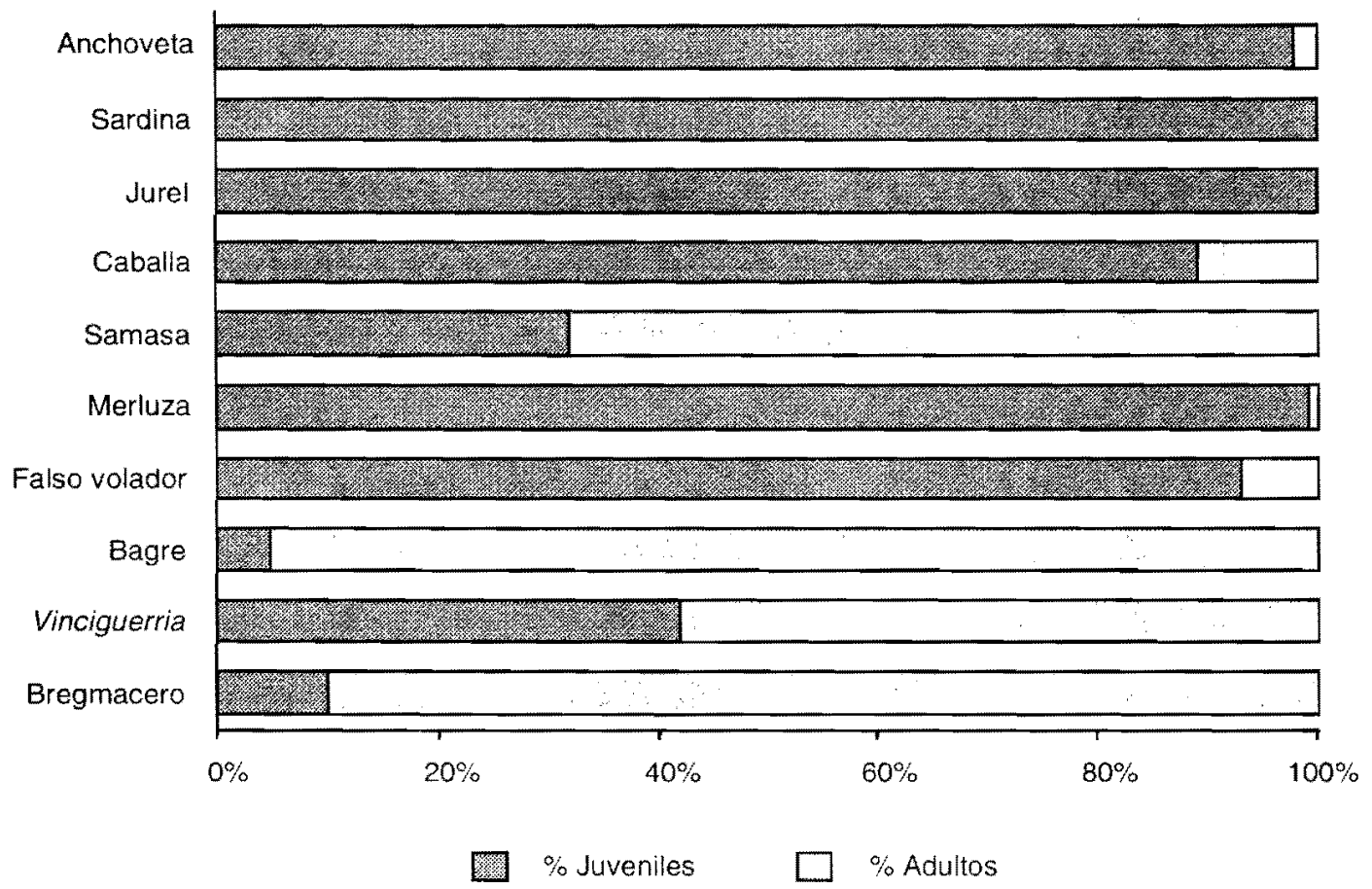

Figura 7. Porcentaje de juveniles de las principales especies marinas en setiembre de 1998.

favorecidos por la incidencia del fuerte evento "E1 Niño 1997-98". Estos reclutamientos fueron detectados en las diferentes fases de "El Niño", especialmente a fines del evento e inicios del post-Niño, como fue observado en setiembre de 1998 (Fig. 7). Esta situación indicó una renovación en las poblaciones de las principales especies pelágicas. En el caso de samasa, bregmacero y bagre (Galeichthys peruvianus), predominaron los adultos, indicando el crecimiento de sus poblaciones durante todo "El Niño 1997-98", casi un ciclo de vida completo durante los 18 meses de duración de las anomalías oceanográficas.

\section{Cambios en el proceso reproductivo}

Durante el evento "El Niño 1997-98", se produjo un desfase en el ciclo reproductivo de la anchoveta, diferente al patrón normal y disminuyó la intensidad del desove (Perea et al., 1998), mientras que en sardina se produjo un efecto contrario, es decir un desove más continuo y se incrementó la intensidad, superando a los patrones normales.

Adicionalmente, en el aspecto reproductivo hay observaciones importantes, como el hecho de haber detectado desovantes en ejemplares juveniles de sardina y caballa. En efecto, se conoce que las sardinas sexualmente maduras son predominantemente de $26-27 \mathrm{~cm}$ de longitud, en esta oportunidad las sardinas sexualmente maduras medían 18,19 y $20 \mathrm{~cm}$, longitudes nunca antes registradas en nuestro medio.

Los actuales rangos de tamaños de sardina, corresponden con los que se encuentran en la sardina de California, las que son sexualmente maduras, en un $50 \%$, a la longitud de $19 \mathrm{~cm}$ según Macewicz (1996). Por otro lado, Parrish et al. (1996) mencionan que, probablemente, tanto la sardina de California, como la sardina de Perú - Chile, tengan un origen común y constituyan una misma especie. Se plantea la interrogante sobre el carácter temporal o permanente en todos los procesos reproductivos.

Debe tenerse en cuenta, que esta ha sido la primera oportunidad para realizar muestreos de tallas juveniles in situ y en una temporada de pre-desove o desove, que ha permitido 

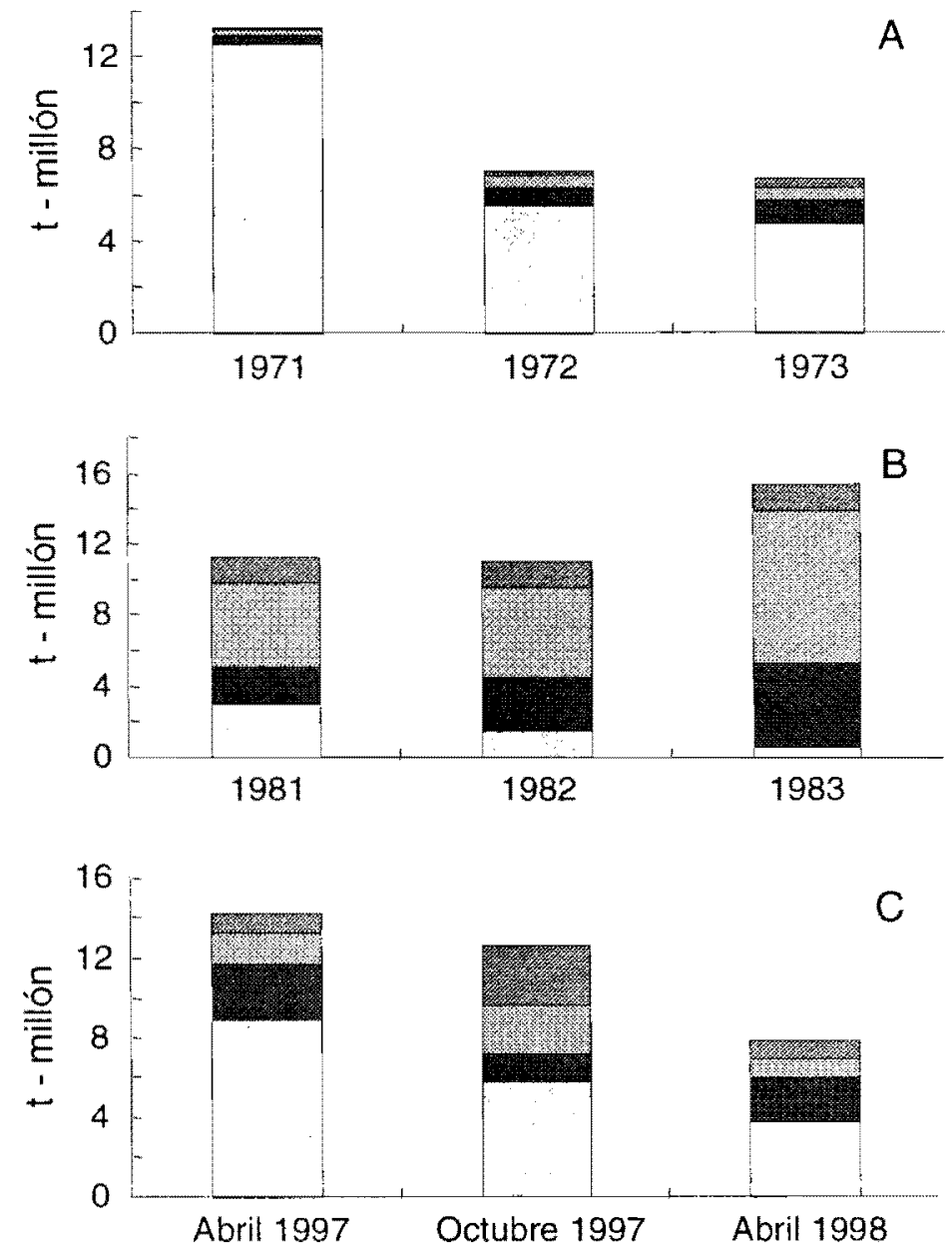

\section{Anchoveta Sardina Jurel Caballa}

Figura 8. Biomasa de especies pelágicas. A) 1971-73. B) 1981-83. C) 1997-98.

subsanar la falta de muestreo en estas tallas y en esta época del año, lo que repercute en la determinación de la longitud al primer desove, según los datos que manejamos.

La otra posibilidad sería la reducción de la tasa de crecimiento, influenciada por variaciones en su alimentación, que se reflejaría en la presencia de sardinas pequeñas, pero de mayor edad, que estarian actualmente desovando.

\section{Cambios en la biomasa de recursos pelágicos}

Durante los eventos "El Niño" se ha registrado una notable disminución de la biomasa de anchoveta y el incremento en las otras especies pelágicas (Fig. 8).

Sin embargo, la magnitud de los impactos, está en función a la intensidad del fenómeno y los antecedentes previos a la acción de "El Niño". En el caso de 1971-73, al inicio del evento, el predominio de anchoveta era casi total, produciéndose un drástico decrecimiento, el mayor en todos los eventos "El Niño" analizados, y al final del evento destaca el incremento de las poblaciones de sardina, jurel y caballa. En "El Niño 198283 ", los antecedentes fueton favorables para los otros recursos pelágicos, que al final del evento representaron $95 \%$ del total de 
biomasa. Finalmente, en "El Niño 1997-98", volvió a disminuir la biomasa de anchoveta (Niquen y Gutiérrez, 1998), mientras que la biomasa de otros pelágicos se incrementaron o tendieron a mantenerse igual durante todo el evento.

En los tres casos analizados, la disminución en la productividad fue general y estuvo marcada por el decrecimiento de la anchoveta. En 1982-83 se observó un incremento en la productividad, debido al ingreso del jurel $(53 \%)$, que es un recurso migratorio $y$ temporal.

\section{Cambios en la pesquería pelágica}

Como consecuencia de las variaciones poblacionales en los principales recursos pelágicos, la pesquería, que normalmente basa sus capturas en el recurso anchoveta, en los tres Niños analizados, se vio seriamente afectada, y tuvo que diversificar las capturas, a sardina, jurel, caballa y samasa. Asimismo, también se aprovechó la disponibilidad de especies foráneas como sardina redonda (Etremeus teres), ayamarca, machete de hebra, sierra (Scomberomorus sierra), perico y otros.

\section{CONCLUSIONES}

Entre Paita y Chimbote $\left(5-10^{\circ} \mathrm{LS}\right)$, se produjeron anomalías térmicas de hasta $8^{\circ} \mathrm{C}$ por encima del promedio, en la etapa de máximo desarrollo de "El Niño 1997. 98 ".

La anchoveta en la región norte-centro mostró una distribución costera, dentro de las 20 millas, con desplazamiento de los cardúmenes hacia el sur de Chimbote y con tendencia a profundizarse por debajo de $\operatorname{los} 10 \mathrm{~m}$.

La estructura por tallas de anchoveta mostró una predominancia de ejemplares adultos, con una baja incidencia de reclutas. En el período post-Niño se detectó un ingreso notable de ejemplares juveniles.
La estructura por tallas de sardina, jurel, caballa y samasa mostró una sincronía en sus buenos reclutamientos, que se hizo evidente durante todo el período "El Niño".

El proceso reproductivo de anchoveta fue afectado en su intensidad y se producen desfases en su época de máxima intensidad, a la espera de encontrar condiciones ambientales más favorables.

Las principales especies pelágicas presentaron un incremento de juveniles, como en algunas especies demersales: falso volador y merluza, indicándonos que la incidencia del evento "El Niño" generaron una rápida renovación en estas poblaciones.

El nivel poblacional de la anchoveta tuvo un notable descenso, mientras que incrementaron otros recursos pelágicos, el falso volador, los peces linterna del género Vinciguerria, el bagre, el pez cinta y los mictófidos, lo que favoreció una explotación pesquera temporal.

\section{LITERATURA CITADA}

Bjerknes. J. 1967. Survey of "El Niño" 1957-58 in its relation to Tropical Pacific Meteorology, Inter-Amer. Thop. Tuna Comm., Rull. 12 (2): $1-42$.

Csirke, J.: R, Gucvara-Carrasco: G. Cárdenas: M. Niquen y A Chipollini. 1996. Situación de los recursos anchoveta (Engrawlis ringens) y sardina (Sardinops sagax) a principios de 1944 y perspectivas para la pesca cn el Perú, con particular referencia a las regiones nonte $y$ centro de la costa peruana. Bol. In.k. Mar PertiCallat 15 (1): 23 pp.

Chirichigno, N. 1978. Nuevas adiciones a la ictiofauna marina del Peru. Inf. Inst. Mar Perti-Callao (46): $1-109$

IMARPE. 1997. Crucero de evaluación hidroacústica de recursos pelágicos; $\mathrm{BIC}$ SNP- 19702 y $\mathrm{BIC}$ HUMBOLDT 9704. In\%. Inst. Mar PeríCallan (127): $91 \mathrm{pp}$.

1998. Crucero de evaluación hidroacústica de recursos pelágicos BIC HUMBOLDT 9803-05 de Tumbes a Tacna. Inf. Inst. Mar Peni-Callao (135): 201 pp.

Macewicz, J. 1996. Pacific Sardine adult reproductive 
parameters. CALCOH Rep. 37: 140-151.

Niquen, M. y M. Gutiérrez. 1998. Variaciones poblacionales y biológicas de los principales recursos pelágicos durante abril 1997 a abril 1998 en el mar peruano. Inf. Inst. Mar PeríCallao (135): 79-90.

Parrish, R.H.; R. Serra \& S. Grant. 1989. The monotypic sardine, Sardina and Sardinops: Their taxonomy, distribution stock structure and zoogeography. Can. J. Fish. Aquat. Sci. (46): 2019-2036.

Perca A.; B. Buitrón y E. Mecklenburg. 1998. Estado reproductivo, fecundidad parcial y frecuencia de desove de la anchoveta peruana a inicios de otoño 1998. Inf. Inst. Mar Peni-Callao (135): 147-152.

Zuta. S.; D. Enfield; J. Valdivia; P. Lagos y C. Blandin. 1974. Aspectos físicos del fenómeno "El Niño" 1972-73. En: Actas, Reunión de Trabajo sobre el fenómeno conocido como "El Niño". FAO Informes de Pesca (185): 3-61.

Zuzunaga. J. 1985. Cambios en el equilibrio poblacional entre la anchoveta (Engraulis ringens) y la sardina (Sardinops sagax) en el sistema do afloramiento frente al Perú. Fn: W. Arntz; A. Landa y J. Tarazona (eds.). "El Niño". su impacto en la fauna marina. Bol. Inst. Ma" Peri-Callao Vol. Extraor.: 107-117. 